\title{
Design of Wideband Waveguide Hybrid with Ultra-Low Amplitude Imbalance
}

Use http://dx.doi.org/10.1109/TTHZ.2015.2502070 when refereeing to the paper. 


\title{
Design of Wideband Waveguide Hybrid with Ultra-Low Amplitude Imbalance
}

\author{
${ }^{1}$ Hawal Rashid, ${ }^{1}$ Vincent Desmaris and ${ }^{1}$ Victor Belitsky \\ ${ }^{2}$ Marcel Ruf, ${ }^{2}$ Thilo Bednorz, ${ }^{2}$ Andreas Henkel \\ ${ }^{1}$ Chalmers University of Technology. Gothenburg, Sweden \\ ${ }^{2}$ Rohde \& Schwarz, GmbH, Germany
}

\begin{abstract}
We present a design and synthesis procedure for a new $90^{\circ}$ waveguide hybrid with ultra-low amplitude imbalance. This novel hybrid design is based on Reed's multiple branch waveguide hybrid. The primary feature of this quadrature hybrid design is the introduction of a controllable ripple in the operational band by, firstly changing the heights of the input and output branches and, secondly, by introducing three waveguide-height discontinuity sections placed symmetrically in the main waveguide. This layout allows ultra-low amplitude imbalance over a wide operational band. At the same time, it permits a greater input/output branch height as compared to the Reed's five-branch waveguide hybrid, which should ease fabrication for short $\mathrm{mm}$ and sub-mm wavelengths.

This design concept is demonstrated through a $90^{\circ}$ waveguide hybrid for the $159-216 \mathrm{GHz}$ band $(30 \%$ fractional bandwidth). Our simulations indicate that the amplitude imbalance of the hybrid is better than $0.2 \mathrm{~dB}$ over the most of the $159-216 \mathrm{GHz}$ band with a phase imbalance better than $\pm 4^{\circ}$. Experimental verification of the hybrid shows excellent agreement with the simulations.
\end{abstract}

Index Terms - Directional coupler, Waveguide hybrids, Millimeter wave devices, Submillimeter wave devices, THz components

\section{INTRODUCTION}

$\mathrm{D}$ IRECTIONAL couplers have been studied since the 1940s, resulting in a variety of design techniques for both waveguide and substrate-based components. The $3 \mathrm{~dB}$ coupler or hybrid is a special case of the directional coupler that provides equal power division/combination and $90^{\circ}$ or 180 degree phase difference between the outputs depending on the design topology. The $3 \mathrm{~dB}$ coupler is one of the basic circuit components for balanced amplifiers [1], sideband separating (2SB) mixers [2],[3],[4], array antennas [5], filers, and more.

There are many design topologies of the substrate-based hybrids with diverse fabrication complexity that offers improved hybrid performance, [1], [6], [7], [8], [9] Nonetheless, the $90^{\circ}$ branch line couplers remain a pertinent research topic even today due to their simplicity and ease of fabrication [10], [11],[12], [13].

In the terahertz frequency spectrum ( $\mathrm{mm}$ wave to $\mathrm{THz}$ ), the

Manuscript submitted on June 17, 2015. The authors are with the Group of Advanced Receiver Development (GARD) at Chalmers University of Technology,Gothenburg,Sweden.(email:Hawal@ chalmers.se) loss of substrate-based transmission lines is significantly larger than that of metal waveguides. Therefore, waveguide couplers are usually preferred. The multiple branch waveguide $90^{\circ}$ hybrid [14], [15] has proven to be very practical for applications such as radio astronomy instrumentation [4], [16], [17],[18]

Reed's periodic multiple branch $90^{\circ}$ waveguide hybrid design [14], [15] allows for very good directivity, return loss and phase imbalance (usually better than $\pm 1^{\circ}$ ) within the operation bandwidth. However, the amplitude imbalance in such components is typically $1 \mathrm{~dB}$ or worse for broadband operation [19]. Furthermore, the maximum amplitude imbalance occurs at the designed center frequency, $f_{0}$. This is an undesired feature in many applications, especially in receiver systems that employ $2 \mathrm{SB}$ layout [16]. Instead, a waveguide structure that allows for controllable ripples in the operating band, similar to substrate based multi-section hybrids [1], [6], [7],[8] is desirable. This issue has partially been addressed in [13] and implemented in ALMA B5 production receiver.

A typical design condition of a waveguide directional coupler is a maximized directivity/isolation [14]. In contrast, we loosen the condition for maximum isolation and modify the hybrid such that interleaved ripples are introduced into the through and coupled signals, thus yielding minimum amplitude imbalance within the operating frequency band. Such a modified hybrid is a trade-off between the controllably degraded return loss and the improved amplitude imbalance.

Specifically, the work presented here concerns the design and synthesis procedure of a novel waveguide $90^{\circ}$ hybrid. The primary feature of this hybrid design is the introduction of a controllable ripple in the operational band by, firstly changing the heights of the input and output branches of the structure and, secondly, by introducing three waveguideheight discontinuity sections placed symmetrically in the main waveguide. This layout not only allows ultra-low amplitude imbalance over a wide operational band, but also significantly improves the return loss at the center frequency. At the same time, the layout permits a greater input/output branch height as compared to Reed's fivebranch waveguide hybrid, which should ease fabrication for short $\mathrm{mm}$ and sub-mm wavelengths.

The design and synthesis procedure were implemented for a demonstrator, a waveguide hybrid operating at 159-216 $\mathrm{GHz}$, which corresponds to approximately $30 \%$ of the 
fractional bandwidth. The performance of the hybrid presented here is significantly improved as compared to recent work [13].

\section{HYBRID DESIGN}

The hybrid presented here is compared with Reed's waveguide hybrid design using the analysis techniques provided by [14] and [15] in order to illustrate the performance improvements and trade-offs.

According to [14], Reed's waveguide hybrid design exhibits optimal performance for an even number of sections. Hence, an eight branch periodic configuration was selected to achieve as low amplitude imbalance as possible. The maximum fractional bandwidth that could be achieved for a maximum amplitude imbalance ripple of $0.2 \mathrm{~dB}$ with minimum $15 \mathrm{~dB}$ return loss and maximum \pm 5 degrees phase imbalance is approximately $30 \%$.

Fig. 1 show the typical cross section of a periodic eightbranch waveguide coupler, where the dashed line indicates the symmetry plane in the structure. The couplers properties can be calculated either with even and odd mode analysis, similar to [15],[20] or by employing matrix analysis methods [17].

The bandwidth is usually determined by the amplitude of the ripple in the operational band. However, in this case, a more comprehensive approach for comparing the performance between the different designs uses a combination of parameters: reflection, isolation, amplitude, and phase imbalance in the operation band i.e., $159-216 \mathrm{GHz}$.

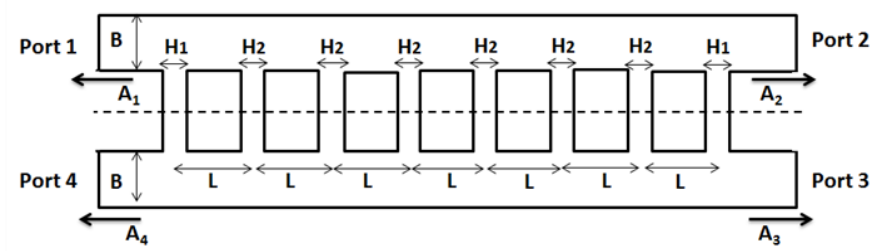

Fig.1. E-plane split block cross section of the eight branch hybrid. The dashed line shows the symmetry plane. $\mathrm{B}$ is the main waveguide height, whereas $\mathrm{H}_{1}$ and $\mathrm{H}_{2}$ are the branch heights.

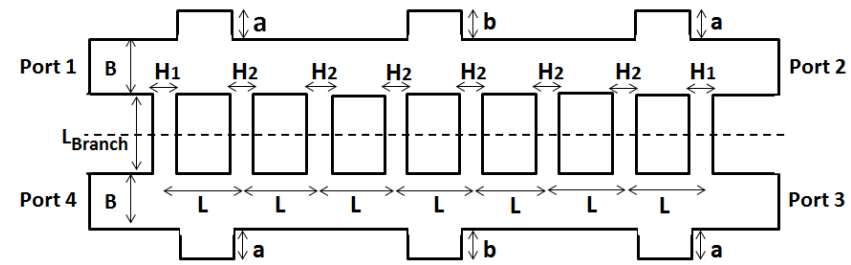

Fig.2. E-plane split block cross section of the proposed eight branch hybrid. The dashed line shows the symmetry plane. $\mathrm{B}$ is the main waveguide height, $\mathrm{H}_{1}$ and $\mathrm{H}_{2}$ are the branch heights, $\mathrm{L}_{\mathrm{Branch}}$ is the branch length, a, and, $\mathrm{b}$, is height extrusions of the main waveguide.

\section{A. Reed's eight branch waveguide hybrid}

A signal with unit amplitude is applied at port 1 of the network in Fig 2. The analytical expression of the vector amplitudes at the outputs, shown in (1), can be determined through the even and odd mode analysis technique described in [15].

$$
\begin{array}{ll}
A_{1}=\frac{1}{2}\left(\Gamma_{e}+\Gamma_{o}\right) & A_{2}=\frac{1}{2}\left(\mathrm{~T}_{e}+T_{o}\right) \\
A_{4}=\frac{1}{2}\left(\Gamma_{e}-\Gamma_{o}\right) & A_{3}=\frac{1}{2}\left(\mathrm{~T}_{e}-\mathrm{T}_{o}\right)
\end{array}
$$

$\Gamma_{e}$ and $\Gamma_{o}$ in equation (1) are reflected even and odd mode vector amplitudes, whereas $\mathrm{T}_{e}$ and $\mathrm{T}_{o}$ are the even and odd mode transmitted vector amplitudes.

Equation (1) can be written in terms of scattering parameters (s-parameters).

$$
\begin{array}{ll}
S_{11}=\frac{1}{2}\left(\Gamma_{e}+\Gamma_{o}\right) & S_{21}=\frac{1}{2}\left(\mathrm{~T}_{e}+T_{o}\right) \\
S_{41}=\frac{1}{2}\left(\Gamma_{e}-\Gamma_{o}\right) & S_{31}=\frac{1}{2}\left(\mathrm{~T}_{e}-\mathrm{T}_{o}\right)
\end{array}
$$

The network (hybrid) in Fig. 2, is divided into several subnetworks, each representing a branch or main waveguide section with geometry (height), $\mathrm{B}, \mathrm{K}_{1} \mathrm{~K}_{2}, \mathrm{H}_{1}$, and $\mathrm{H}_{2}$ respectively. The ABCD matrices for each subnet are then sequentially multiplied as shown below.

$$
\begin{aligned}
& {\left[\begin{array}{ll}
A & B \\
C & D
\end{array}\right]_{e} }=\left[\begin{array}{cc}
1 & j H_{1} P \\
0 & 1
\end{array}\right]\left[\begin{array}{cc}
0 & j K_{1} t \\
j t / K_{1} & 0
\end{array}\right]\left[\begin{array}{cc}
1 & j H_{2} P \\
0 & 1
\end{array}\right]\left[\begin{array}{cc}
0 & j t \\
j t & 0
\end{array}\right] \\
& \times\left[\begin{array}{cc}
1 & j H_{2} P \\
0 & 1
\end{array}\right]\left[\begin{array}{cc}
0 & j t \\
j t & 0
\end{array}\right]\left[\begin{array}{cc}
1 & j H_{2} P \\
0 & 1
\end{array}\right]\left[\begin{array}{cc}
0 & j K_{2} t \\
j t / K_{2} & 0
\end{array}\right] \\
& \times\left[\begin{array}{cc}
1 & j H_{2} P \\
0 & 1
\end{array}\right]\left[\begin{array}{cc}
0 & j t \\
j t & 0
\end{array}\right]\left[\begin{array}{cc}
1 & j H_{2} P \\
0 & 1
\end{array}\right]\left[\begin{array}{cc}
0 & j t \\
j t & 0
\end{array}\right] \\
& \times\left[\begin{array}{cc}
1 & j H_{2} P \\
0 & 1
\end{array}\right]\left[\begin{array}{cc}
0 & j K_{1} t \\
j t / K_{1} & 0
\end{array}\right]\left[\begin{array}{cc}
1 & j H_{1} P \\
0 & 1
\end{array}\right] \\
& {\left[\begin{array}{ll}
A & B \\
C & D
\end{array}\right]_{o}=\left[\begin{array}{cc}
A_{e}\left(-\frac{1}{P}\right) & B_{e}\left(-\frac{1}{P}\right) \\
C_{e}\left(-\frac{1}{P}\right) & D_{e}\left(-\frac{1}{P}\right)
\end{array}\right] }
\end{aligned}
$$

where $K_{1}=(B+a) / B$ and $K_{2}=(B+b) / B . B$ is the main waveguide height as shown in Fig.2, $t=\tan (\theta), P=$ $\tan \left(\frac{\theta}{2}\right), \theta$ is the electrical length in the waveguide i.e., $\theta=$ $\pi l \sqrt{\left(2 / \lambda_{0}\right)^{2}-(1 / A)^{2}}, \mathrm{~A}$ is main waveguide width and $\lambda_{0}$ is the free-space wavelength.

The even and odd mode reflection and transmission terms can be expressed as follows

$$
\begin{aligned}
\Gamma_{i} & =\frac{1}{2} \frac{(A-D)+(B-C)}{A+B+C+D} \\
\mathrm{~T}_{i} & =\frac{1}{2}\left(\frac{2}{A+B+C+D}\right)
\end{aligned}
$$

where the index $i$, is either, e, for even mode or, o, for odd mode.

The height of the main waveguide sections where the extrusions are placed $\left(\mathrm{K}_{1}\right.$ and $\mathrm{K}_{2}$, see equation 3 ) for Reed's waveguide hybrid design is equal to unity i.e., no extrusions in the main waveguide sections $(a=b=0)$. Furthermore, the waveguide height of the first and last branch $\left(\mathrm{H}_{1}\right)$ must remain smaller than the other branch heights $\left(\mathrm{H}_{2}\right)$. These conditions must be satisfied in order to realize perfect directivity/isolation. It is also found that (3) is greatly simplified when these conditions are met, resulting in analytical expressions for $\mathrm{H}_{1}$ and $\mathrm{H}_{2}$. The analytic expressions for the branch heights can be found in [15]. The performance of Reed's waveguide hybrid design can be determined from equations (2)-(4). The calculated S- 
parameters show that port 1 is matched and no power leaks into port 4 , i.e., perfect isolation as expected. The amplitude imbalance of Reed's waveguide hybrid design is depicted in Fig. 3. Furthermore, the matrix calculations reveals the relationship between the bandwidth and the amplitude imbalance; specifically, the wider the bandwidth the greater the amplitude imbalance at the center frequency. This property limits the usefulness of the hybrid in wideband applications, for example in sideband separating receivers [8].

Beside the issue with the amplitude imbalance, there is yet a much more severe issue, namely the geometry of Reed's eight branch waveguide hybrid. The input branch height $\left(\mathrm{H}_{1}\right)$ for an eight branch coupler in, e.g., WR5 waveguide is $67 \mu \mathrm{m}$ according to the table given in [15]. An input branch height of $67 \mu \mathrm{m}$ is not practical either for conventional milling machines or for modern computer numerical control (CNC) milling as the required aspect ratio of the mill becomes too large. Therefore, for practical considerations, a five branch hybrid is more feasible since the input branch height then becomes $131 \mu \mathrm{m}$. However, using a 5 branch coupler would degrade the amplitude imbalance by approximately $15 \%$.

A theoretical comparison of amplitude imbalance with respect to the normalized guide wavelength for waveguide hybrid with different number of branches and bandwidth are shown in Fig.3. The normalized guide wavelength, $\lambda_{\mathrm{g} 0} / \lambda_{\mathrm{gs}}$, is defined as follows:

$$
\begin{gathered}
\lambda_{g s}=\frac{1}{\sqrt{\frac{1}{\lambda_{0}^{2}}-\frac{1}{4 A^{2}}}} \\
\lambda_{g 0}=2 \frac{\lambda_{g, H} \lambda_{g, L}}{\lambda_{g, H}+\lambda_{g, L}}
\end{gathered}
$$

where $\lambda_{\mathrm{g} 0}$ is the guide wavelength at the center of the operational bandwidth, whereas $\lambda_{\mathrm{g}, \mathrm{H}}$ and $\lambda_{\mathrm{g}, \mathrm{L}}$ are the shortest and longest guide wavelengths in the operational bandwidth. $\lambda_{\mathrm{gs}}$ is guide wavelength with respect to free-space wavelength, $\lambda_{0}$ and main waveguide width, $\mathrm{A}$.

Normally, the fractional bandwidth is calculated by dividing the difference between the highest and lowest frequency with the center frequency, that is:

$$
B W=\frac{f_{H}-f_{L}}{f_{0}}
$$

The same is not true for guide wavelength, that is:

$$
B W \neq \frac{\lambda_{g, L}-\lambda_{g, H}}{\lambda_{g 0}}
$$

The guide wavelength is a function of waveguide geometry parameters and frequency, as shown in the equation (6). Therefore, the frequency needs to be written with respect to both guide wavelength and the geometry. In this case, equation (6) gives

$$
B W=\frac{\sqrt{\frac{4}{\lambda_{g, H}^{2}}+\frac{1}{A^{2}}}-\sqrt{\frac{4}{\lambda_{g, L}^{2}}+\frac{1}{A^{2}}}}{\sqrt{\frac{4}{\lambda_{g 0}^{2}}+\frac{1}{A^{2}}}}
$$

Equation (8) could be used to calculate the fractional bandwidth in Figs. 3-8.

The solid black line corresponds to the proposed 8 branch hybrid (described in next section). The dashed gray line corresponds to Reed's eight branch waveguide hybrid, where the bandwidth is defined with respect to the maximum ripple of the proposed design. The dashed gray line with solid triangles is Reed's eight branch waveguide hybrid, where the bandwidth between two points of zero amplitude imbalance is equivalent to the bandwidth of the proposed design. The dashed gray line with solid dots corresponds to Reed's five branch waveguide hybrid equivalent to Reed's eight branch waveguide hybrid.

Detailed synthesis of Reed's waveguide hybrid design can be found in [14],[15].

\section{B. Modified eight-branch waveguide hybrid}

In order to generate ripples in the operation band, the condition for perfect directivity needs to be relaxed. This means that small reflections inside the waveguide hybrid need to be deliberately created. Trying to solve this problem analytically by arbitrary placing, e.g., modulation of the waveguide height similar to Fig. 2 would be very tedious and difficult process, since (3) grows rapidly in complexity.

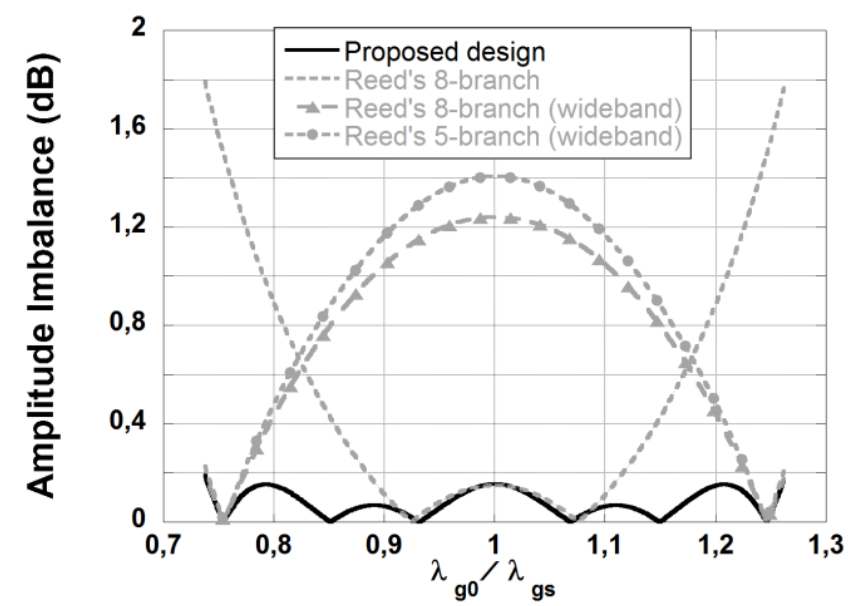

Fig. 3. Theoretical comparison of amplitude imbalance for waveguide hybrids with different numbers of branches and bandwidths.

Different approaches can be used to introduce mismatch in the hybrid. The number of height extrusion sections can be minimized by imposing the condition that the reciprocity of network must be preserved. This means that the mismatches need to be symmetrically located within the waveguide hybrid layout.

A desirable step in the design process is to increase the branch height so that $\mathrm{CNC}$ milling could be used.

Therefore, a solution to (2)-(4) where $\mathrm{H}_{1}$ is substantially larger than $67 \mu \mathrm{m}$ was employed, which introduces a small mismatch. Further mismatch can be introduced by changing 
the geometry (height) of the main waveguide sections between the first and second branches and between the $4^{\text {th }}$ and $5^{\text {th }}$ branches as illustrated in Fig. 2. At these oversized sections, a TE01 mode at approximately $200 \mathrm{GHz}$ could be excited. However, the excited wave would turn into a strong evanescent wave as soon as it enters the main waveguide with height, B. Hence, this effect could be neglected.

\section{Synthesis of the modified eight-branch waveguide hybrid}

The synthesis of the proposed waveguide hybrid can be carried out through an iterative four step process as described below.

I. Set $\mathrm{K}_{1}$ and $\mathrm{K}_{2}$ to unity, and $\mathrm{H}_{1}>\mathrm{H}_{2} . \mathrm{H}_{1}$ is typically 15 $30 \%$ larger than $\mathrm{H}_{2}$.

II. Use (2)-(4) to find the values of $\mathrm{H}_{1}$ and $\mathrm{H}_{2}$, which results in the required coupling for the given bandwidth.

III. Let $\mathrm{K}_{1}$ remain unchanged (unity for initial run) and sweep $K_{2}$ in order to find the proper amplitude ripple in the operation bandwidth.

IV. Let $\mathrm{K}_{2}$ remain unchanged and sweep $\mathrm{K}_{1}$ in order to find the proper amplitude ripple in the operation bandwidth.

V. Repeat step II-IV until optimal amplitude imbalance are reached, which is typically a tradeoff between return loss and phase imbalance vs. bandwidth.

The $\mathrm{T}$-sections between the branch and main waveguide need to be corrected, i.e., adjusting $\mathrm{K}_{1}, \mathrm{~K}_{2}, \mathrm{H}_{1}$ and $\mathrm{H}_{2}$ before running electromagnetic structure simulator. The correction procedure is described in [21] and [14].

The amplitude imbalance after the synthesis process as described above is $1.2 \mathrm{~dB}$ better than Reed's waveguide hybrid design, which is a substantial improvement.

\section{The characteristics of the modified eight-branch waveguide hybrid}

The characteristics of the proposed hybrid given by the matrix based calculations are illustrated in Figs. $4-7$. Furthermore, extrusion parameters, $a$, and $b$, in Figs. 4-7 are normalized to main waveguide height, $B$, and plotted with respect to the normalized guide wavelength, $\lambda_{\mathrm{g} 0} / \lambda_{\mathrm{gs}}$. The amplitude in Fig.4 shows the special case [13], were the height extrusion, $\mathrm{b} / \mathrm{B}\left(\mathrm{K}_{2}\right)$ are increased with steps of 0.052 (the step corresponds to approximately $33 \mu \mathrm{m}$ for a WR5 waveguide) while, $a / B$, is held constant at zero i.e., $\mathrm{K}_{1}=1$. Figure 4 and 5 show that the larger the height extrusion (larger $\mathrm{K}_{1}$ and/or $\mathrm{K}_{2}$ ), the larger the ripple amplitude becomes, thereby increasing the bandwidth of the waveguide hybrid. However, the surface plots in Fig. 6 and Fig. 7, show that the gain in bandwidth and performance enhancement of the amplitude imbalance comes at the cost of further impedance mismatch and slightly degraded phase balance. Similarly, the introduced impedance mismatch due to increasing the main waveguide heights $\mathrm{K}_{1}$ and $\mathrm{K}_{2}$ (by increasing the extrusions, a, and b in Fig. 2) deteriorates the return loss and isolation as can be observed in Fig. 7. The average coupling value of $3 \mathrm{~dB}$ for the hybrid will be slightly shifted due to the degraded return loss (see Fig. 8).
Table I summarizes performance of the waveguide hybrid in [13], five and eight branch Reed's waveguide hybrid design, and this work.

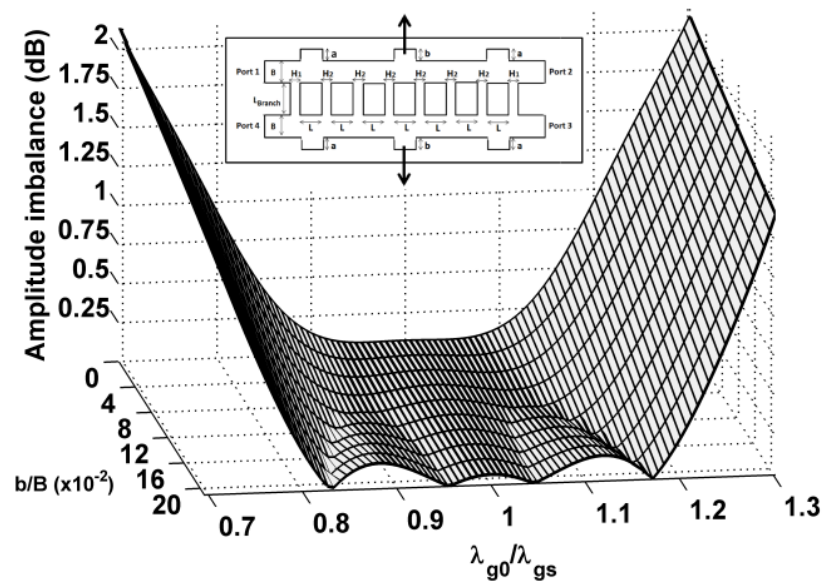

Fig. 4. Calculated amplitude imbalance performance of the proposed hybrid with respect to different main waveguide height extrusion parameter, $b\left(\mathrm{~K}_{2}\right)$ and frequency while $a=0$.

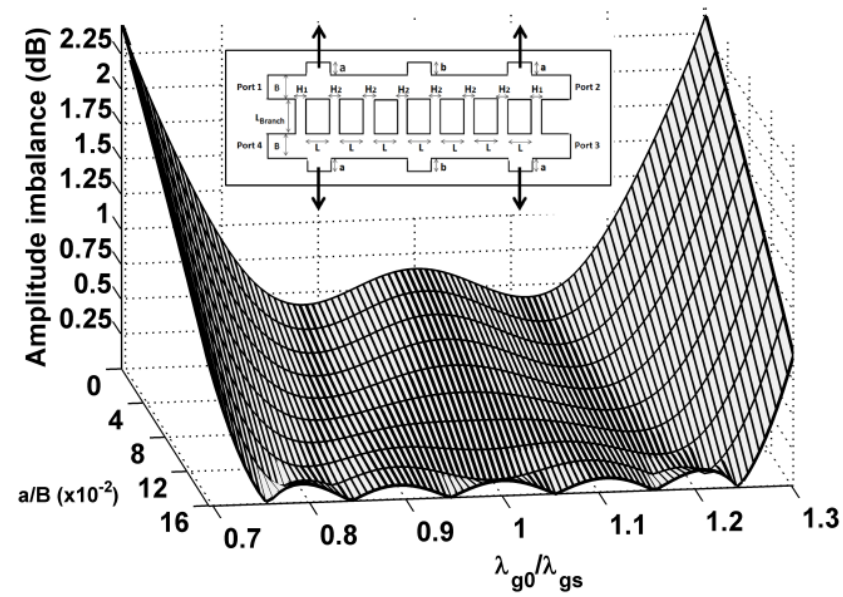

Fig. 5. Calculated amplitude imbalance performance of the proposed hybrid with respect to different main waveguide height extrusion parameter, $a\left(\mathrm{~K}_{1}\right)$ and frequency, $b / B=0.206$.

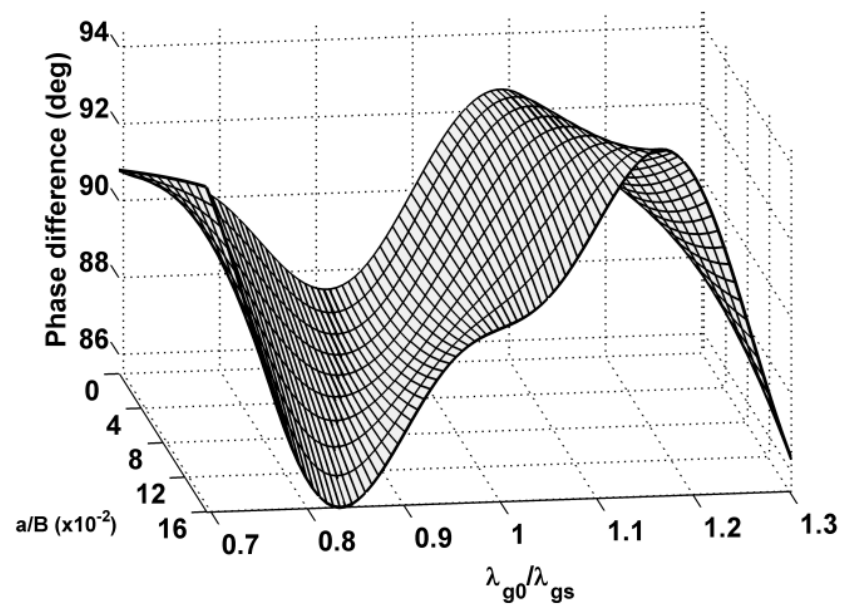

Fig. 6. Calculated phase difference performance of the proposed hybrid with respect to different main waveguide height extrusion parameter, $a\left(\mathrm{~K}_{1}\right)$ and frequency, $b / B=0.206$, see insert in Fig. 5. Notice that the scale of vertical axis is on the order of a few degrees only. 


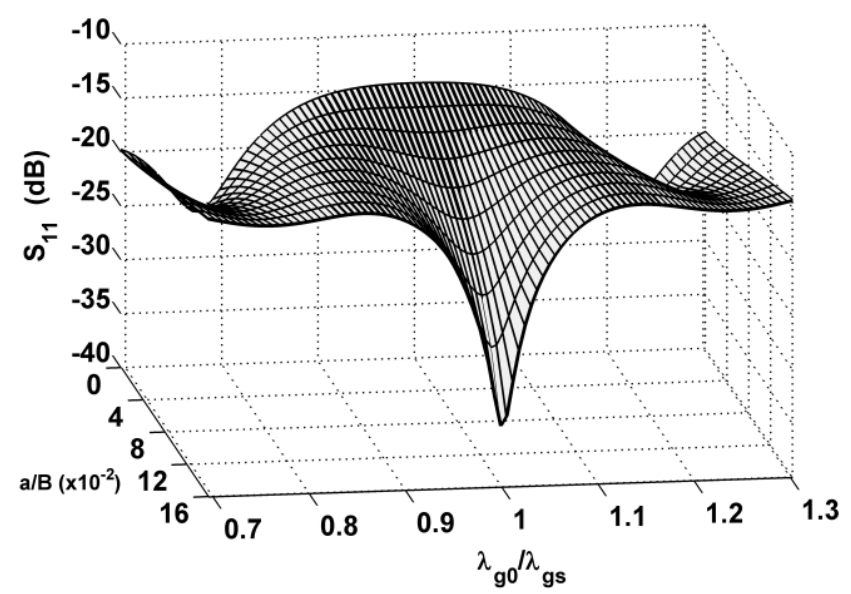

Fig. 7. The reflected portion of the pilot signal, $S_{11}$ vs. height of main waveguide height extrusion parameter, $a$ (different $\mathrm{K}_{1}$ ) and frequency, $b / B=0.206$, see insert in Fig. 5. The response of the isolated port, $\mathrm{S}_{41}$, is identical to due $\mathrm{S}_{11}$ to symmetry.

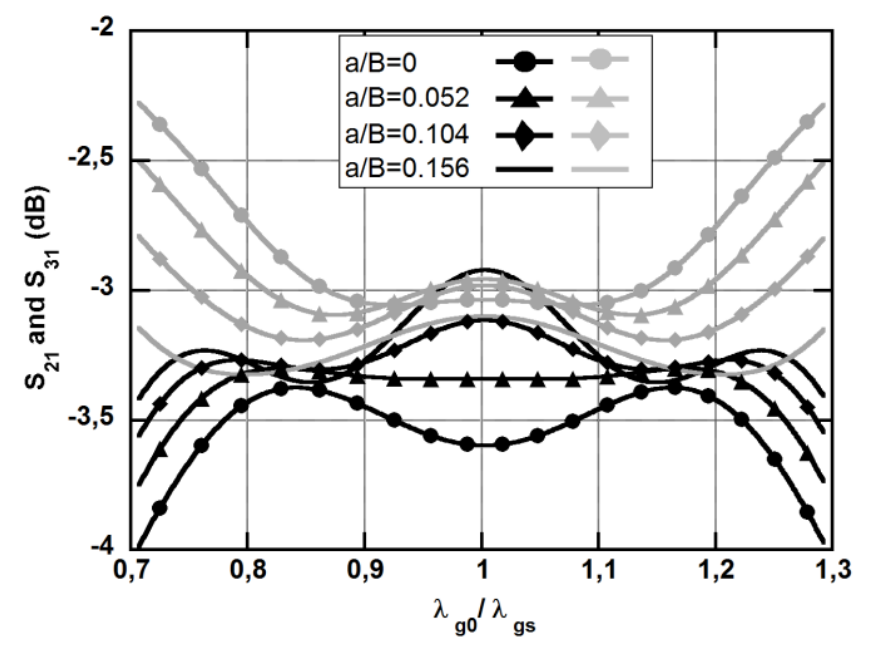

Fig. 8. The calculated through, S21 and coupled, S31 for the proposed hybrid with respect to different main waveguide height extrusion $a$ (different $\mathrm{K}_{1}$ ) and frequency, while $b / B=0.206$.

TABLE I

WAVEGUIDE HYBRID PARAMETERS FOR DIFFERENT DESIGNS WITHOUT CORRECTIONS ( $\Delta \mathrm{A}$ IS THE AMPLITUDE IMBALANCE, $\Delta \varphi$ IS THE PHASE IMBALANCE, AND RL IS THE RETURN LOSS)

\begin{tabular}{ccccccc}
\hline \hline Paper & $\begin{array}{c}\mathrm{H}_{1} \\
/ \mathrm{H}_{2}\end{array}$ & {$[\mathrm{a}, \mathrm{b}] \mu \mathrm{m}$} & $\begin{array}{c}\mathrm{BW} \\
\%\end{array}$ & $\begin{array}{c}\mathrm{RL}(\mathrm{dB}) \\
{\left[\max , f_{0}\right]}\end{array}$ & $\begin{array}{c}\Delta \mathrm{A} \\
\mathrm{dB}\end{array}$ & $\Delta \varphi$ \\
\hline $\begin{array}{c}5 \text { branch } \\
\text { Reed's } \\
\text { waveguide } \\
\text { hybrid }\end{array}$ & 0.52 & {$[0,0]$} & 30 & $>[25,25]$ & 1.4 & $\pm 0.2^{\circ}$ \\
$\begin{array}{c}8 \text { branch } \\
\text { Reed's }\end{array}$ & & & & & & \\
$\begin{array}{c}\text { waveguide } \\
\text { hybrid }\end{array}$ & 0.52 & {$[0,0]$} & 30 & $>[25,25]$ & 1.2 & $\pm 0.2^{\circ}$ \\
$\begin{array}{c}\text { [13] } \\
\text { This work }\end{array}$ & 1.33 & {$[0,80]$} & 20 & $>[16,16]$ & 0.15 & $\pm 2.3^{\circ}$ \\
& & & & & & \\
\end{tabular}

E. Design Implementation for the 159-216 GHz operation band

The design procedure described in subsection $\mathrm{C}$ was implemented for the frequency range of 159-216 GHz. The selected band is within the existing in-house Vector Network Analyzer (VNA) extension modules, which operate from $140 \mathrm{GHz}$ to $220 \mathrm{GHz}$ in order to characterize the hybrid performance.
The performance of the proposed hybrid was simulated in the 3D electromagnetic structure simulator Keysight EMPro. The design parameters $\left(\mathrm{H}_{1}\right.$ and $\left.\mathrm{H}_{2}\right)$ calculated from theoretical analysis [14], [15] were slightly tuned in order to approximately match the simulated performance with the matrix based performance of (2)-(4). The amplitude imbalance is slightly larger for the simulated hybrid as compared to the matrix based calculations of the hybrid. Furthermore, there is a very small asymmetry in the amplitude imbalance (Fig. 11). The reason for the asymmetry is that branch lengths, $\mathrm{L}_{\mathrm{Branch}}$ were not exactly corresponding between the EM simulation and in the fabricated hybrid. For these reasons, in Fig. 11 only three ripples are seen. On the other hand, this is just a matter of a small EM-optimization of the geometry. The waveguide hybrid dimensions (see. Fig.2) are summarized in table 2. Notice that the smallest dimension in this eight branch hybrid design is larger than its counterpart in the five branch hybrid. The waveguide hybrid was fabricated using an Eplane split block assembly (see Fig. 9) in our in-house workshop with a KERN CNC milling machine, which has \pm $2 \mu \mathrm{m}$ accuracy. Table 2 gives the dimensions.

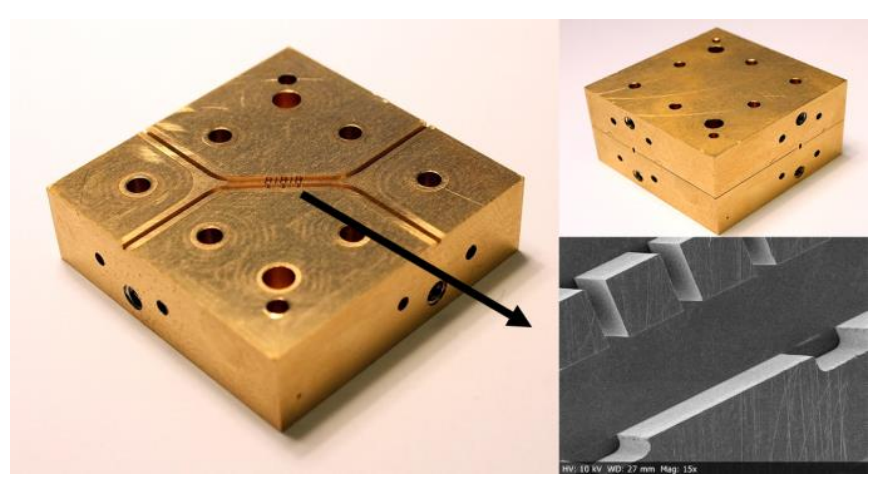

Fig. 9. The picture in the upper right corner show the assembled hybrid, whereas the picture to left show one the E-plane split-block piece. The SEM picture to the lower right corner shows a part of the hybrid.

A tolerance analysis was made using Keysight EMPro, where both Reed's waveguide hybrid design and the presented hybrid were compared. The machining tolerances of the two hybrids are very similar. According to the simulations, the main waveguide height $\mathrm{B}$, and branch length, $\mathrm{L}_{\mathrm{Branch}}$ has a machining tolerance of $\pm 5 \mu \mathrm{m}$ or better, thus a small deviation such as $\pm 2 \mu \mathrm{m}$ has negligible effect on the hybrid performance. Furthermore, the simulations also showed that variations of $\pm 5 \mu \mathrm{m}$ in the main waveguide height extrusions $\mathrm{K}_{1}$ and $\mathrm{K}_{2}(a$, and $b$ ) has negligible effect. However, when the entire branch height $\left(\mathrm{H}_{\mathrm{n}}\right)$, deviates simultaneously by the same amount from the design value, this would result in approximately $0.1 \mathrm{~dB}$ imbalance (for $\pm 2 \mu \mathrm{m}$ ).

\section{TABLE II}

DIMENSIONS OF THE MEASURED WAVEGUIDE HYBRID. ALL DIMENSIONS ARE IN MICROMETERS

\begin{tabular}{ccccccc}
\hline \hline $\mathrm{A} / 2=\mathrm{B}$ & $\mathrm{H}_{1}$ & $\mathrm{H}_{2}$ & Lbranch & $\mathrm{L}$ & $\mathrm{a}$ & $\mathrm{b}$ \\
\hline 630 & 154 & 137 & 404 & 524 & 100 & 130
\end{tabular}

\section{MEASUREMENTS}

The demonstrator waveguide hybrid was fabricated using an E-plane split block assembly. Table 2 gives the dimensions. 
The hybrid was measured with a four port Vector Network Analyzer (VNA) with a Rohde \& Schwarz ZC220 140-220 $\mathrm{GHz}$ transmitter and receiver modules. The ports of the DUT are at right angles to each other, which require a substantial rotation of the transmitter/receiver modules for measuring the coupled port (see Fig .10). Because of the rotation of the modules between measurements, the VNA cables are flexed, which causes errors in the phase measurements. For this reason, the UOSM (unknown through, offset short circuit, short circuit, and match load) calibration technique was used together with a built-in VNA calibration function, which allows re-using or re-measuring any of used calibration standards in the UOSM. The unknown standard, which is the hybrid itself, was remeasured in order to improve the phase accuracy after moving the modules.

As the hybrid is a reciprocal four-port device, only through, coupled and isolated S-parameters need to be measured in order to fully characterize the hybrid. The reflection coefficient is obtained in any of these measurements. In the first measurement, a test signal is applied at Port 1 (see Fig.2). The output signals are measured at Port 2 (through) and input Port 1 (reflected signal) while the remaining ports are terminated with waveguide matched loads from the calibration kit.

Subsequently, Ports 1 and 3 (coupled) were connected and measured (after recalibration of the unknown through standard) while the remaining ports are terminated. Finally, Ports 1 and 4 were connected and measured while the through and coupled ports were terminated. In order to account for the conductive loss in the connecting waveguides (between the calibration planes at the output of the extension modules to the input of the hybrid structure), a two port fixture with the exact shape and length as the hybrid through path was fabricated. The S-parameters from the fixture were measured and used to de-embed the losses (found to be $0.015 \mathrm{~dB} / \mathrm{mm}$ ) of the connecting waveguides from the measured hybrid S-parameters. The insertion loss of the hybrid itself is approximately $0.25 \mathrm{~dB}$ (Fig.11).

Fig. 11 and 12 show the measured performance of the hybrid with eight branches and impedance steps in the main waveguide as depicted in Fig.2. The measured factional bandwidth is approximately $29.5 \%$.

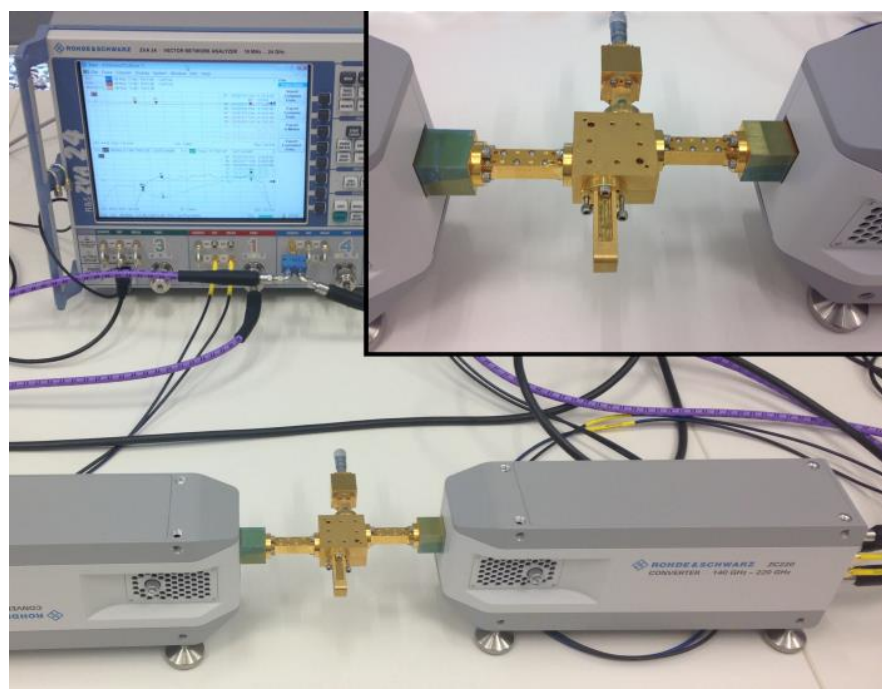

Fig. 10. A picture of the measurement setup, including the $140-220 \mathrm{GHz}$ extension modules with the waveguide hybrid connected. The insert show that the remaining ports are terminated with matched loads during the measurements.

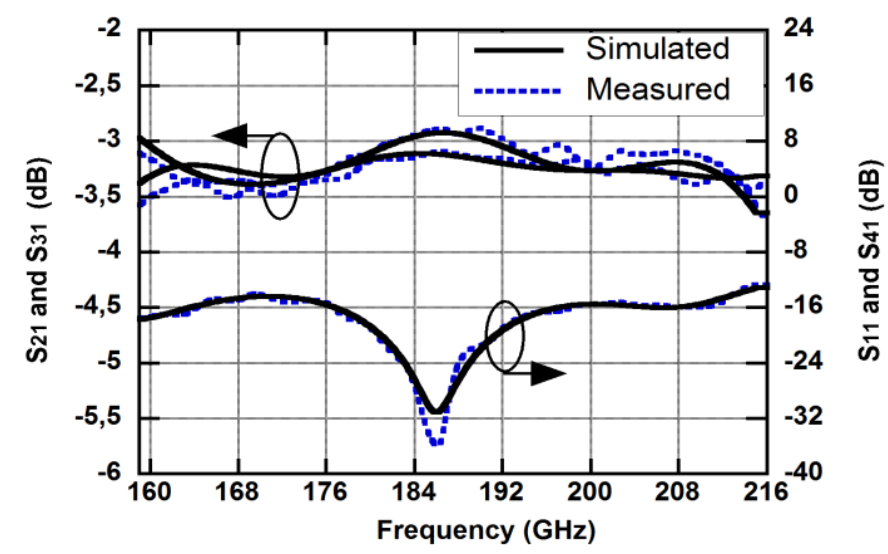

Fig. 11. Measured and simulated through, coupled, reflection and isolation of the proposed design.

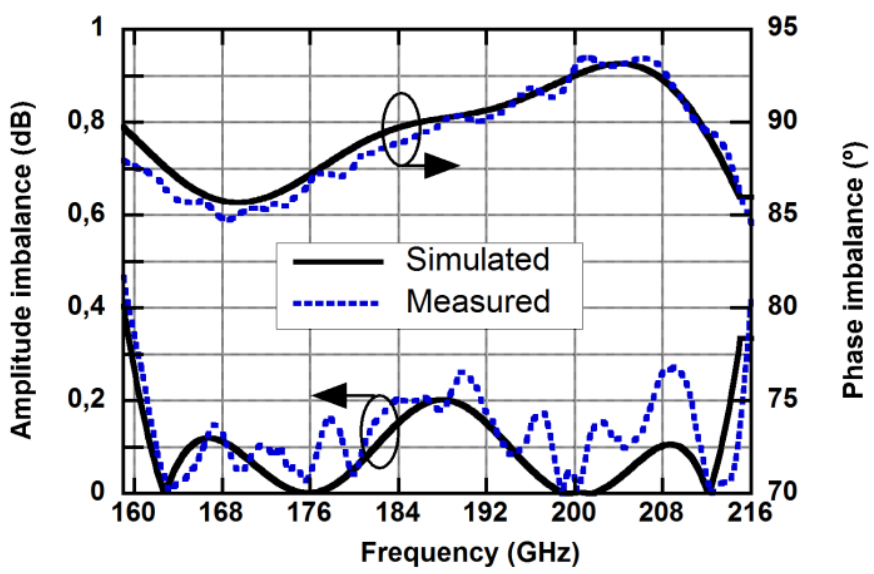

Fig. 12. Measured and simulated amplitude and phase difference between the output ports of the proposed design.

The amplitude imbalance in Fig. 12 is slightly larger for the simulated hybrid as compared to the matrix based analysis, since the geometrical parameters of simulated hybrid was slightly different in order get the correct coupling; the difference is approximately $0.05 \mathrm{~dB}$. Furthermore, there is a very small asymmetry in the amplitude imbalance. The reason for the asymmetry is that, for ease of fabrication, the branch length, $\mathrm{L}_{\mathrm{Branch}}$, was not adjusted.

The slight discrepancy between the simulated and measured data is mostly due to that the branch heights was fabricated $5 \mu \mathrm{m}$ too long and to some small extent because the rounding of the main waveguide extrusions ( $a$, and $b$ ) was not included in the simulation. However, the measured performance of the hybrid still shows excellent agreement with simulations.

\section{CONCLUSIONS}

We have presented a design and synthesis procedure for a new $90^{\circ}$ waveguide hybrid. The hybrid design is based on the multiple branch waveguide design of Reed [3].

The primary feature of this hybrid design is the introduction of a controllable ripple in the operational band by, firstly changing the heights of the input and output branches of the structure and, secondly, by introducing three waveguide- 
height discontinuity sections placed symmetrically in the main waveguide. This layout allows ultra-low amplitude imbalance over a wide operational band. At the same time, it permits a greater input/output branch height as compared to Reed's five-branch waveguide hybrid, which should ease fabrication for short $\mathrm{mm}$ and sub-mm wavelengths. This design concept could be used for waveguide hybrids up to several THz by using, e.g., microfabrication techniques [22], [23], [24]. Our simulations indicate that the amplitude imbalance of the designed hybrid is better than $0.2 \mathrm{~dB}$ over the most of the $159-216 \mathrm{GHz}$ band with phase imbalance better than $\pm 4^{\circ}$. Experimental verification of the hybrid shows excellent agreement with the simulations.

\section{ACKNOWLEDGMENT}

The authors would like to thank Sven-Erik Ferm and Mathias Fredrixon for the hybrid fabrication. The authors would also like to thank Alexey Pavolotsky for fruitful discussions.

\section{References}

[1] I. Malo-Gomez, J. D. Gallego-Puyol, C. Diez-Gonzalez, I Lopez-Fernandez, and C. Briso-Rodriguez, "Cryogenic Hybrid Coupler for Ultra-Low-Noise Radio Astronomy Balanced Amplifiers," Microwave Theory and Techniques, IEEE Transactions on, vol. 57, pp. 3239-3245, 2009.

[2] P. J. Sobis, A. Emrich, and J. Stake, "A Low VSWR 2SB Schottky Receiver," Terahertz Science and Technology, IEEE Transactions on, vol. 1, pp. 403-411, 2011.

[3] J. W. Kooi, R. A. Chamberlin, R. Monje, B. Force, D. Miller, and T. G. Phillips, "Balanced Receiver Technology Development for the Caltech Submillimeter Observatory," Terahertz Science and Technology, IEEE Transactions on, vol. 2, pp. 71-82, 2012.

[4] B. Billade, O. Nystrom, D. Meledin, E. Sundin, I. Lapkin, M Fredrixon, et al., "Performance of the First ALMA Band 5 Production Cartridge," Terahertz Science and Technology, IEEE Transactions on, vol. 2, pp. 208-214, 2012.

[5] L. Kun-Han, L. Keng-Chih, L. Shih-Kai, and L. Yi-Cheng, "Wideband circularly-polarized aperture antenna arrays utilizing UWB directional coupler," in Antennas and Propagation Society International Symposium (APSURSI), 2012 IEEE, 2012, pp. 1-2.

[6] E. G. Cristal and L. Young, "Theory and Tables of Optimum Symmetrical TEM-Mode Coupled-Transmission-Line Directional Couplers," Microwave Theory and Techniques, IEEE Transactions on, vol. 13, pp. 544-558, 1965.

[7] C. Jeong-Hoon, H. Hee-Yong, and Y. Sang-Won, "A design of wideband 3-dB coupler with $\mathrm{N}$-section microstrip tandem structure," Microwave and Wireless Components Letters, IEEE vol. 15, pp. 113-115, 2005.

[8] H. Rashid, D. Meledin, V. Desmaris, A. Pavolotsky, and V. Belitsky, "Superconducting 4-8 GHz Hybrid Assembly for 2SB Cryogenic THz Receivers," Terahertz Science and Technology, IEEE Transactions on, vol. 4, pp. 193-200, 2014.

[9] J. Hesler and A. W. Lichtenberger, "THz Waveguide Couplers Using Quartz Micromachining," presented at the 21ST INTERNATIONAL SYMPOSIUM ON SPACE TERAHERTZ TECHNOLOGY, OXFORD, 2010.

[10] N. A. Sutton, J. M. Oliver, and D. S. Filipovic, "Wideband 18$40 \mathrm{GHz}$ Surface Micromachined Branchline Quadrature Hybrid," Microwave and Wireless Components Letters, IEEE, vol. 22, pp. 462-464, 2012.

[11] T. Ching-Wen, T. Chien-Tai, and H. Ko-Cheng, "Design of Wide Passband Microstrip Branch-Line Couplers With Multiple Sections," Components, Packaging and Manufacturing Technology, IEEE Transactions on, vol. 4, pp. 1222-1227, 2014. S. Gomha, E. S. M. El-Rabaie, and A. A. T. Shalaby, "Optimizing the performance of branch-line couplers using open ended stubs," in Computing, Electrical and Electronics Engineering (ICCEEE), 2013 International Conference on, 2013, pp. 363-367.
[13] H. Rashid, D. Meledin, V. Desmaris, and V. Belitsky, "Novel Waveguide $3 \mathrm{~dB}$ Hybrid With Improved Amplitude Imbalance," Microwave and Wireless Components Letters, IEEE, vol. 24, pp. 212-214, 2014

[14] G. Matthaei, L. Young, and E. M. T. Jones, Microwave Filters, Impedance-Matching Networks, and Coupling Structures: Dedham, MA: Artech House, 1980.

[15] J. Reed, "The Multiple Branch Waveguide Coupler," IEEE Transactions on Microwave Theory and Techniques, vol. 6, pp. 398-403, 1958.

[16] A. website. www.almaobservatory.org.

[17] H. Andoh, S. Asayama, H. Ogawa, N. Mizuno, A. Mizuno, T. Tsukamoto, et al., "Numerical Matrix Analysis for Performances of Wideband $100 \mathrm{GHz}$ Branch-Line Couplers," International Journal of Infrared and Millimeter Waves, vol. 24, pp. 773-788, 2003/05/01 2003.

[18] D. Meledin, A. Pavolotsky, V. Desmaris, I. Lapkin, C. Risacher, V. Perez, et al., "A 1.3-THz Balanced Waveguide HEB Mixer for the APEX Telescope," Ieee Transactions on Microwave Theory and Techniques, vol. 57, pp. 89-98, Jan 2009.

[19] S. Srikanth and A. Kerr, "Waveguide quadrature hybrids for ALMA receivers," ALMA Memo \#343, 2001.

[20] J. Reed and G. J. Wheeler, "A Method of Analysis of Symmetrical Four-Port Networks," Microwave Theory and Techniques, IRE Transactions on, vol. 4, pp. 246-252, 1956. 1965.

V. Desmaris, D. Meledin, A. Pavolotsky, R. Monje, and V. Belitsky, "All-metal micromachining for the fabrication of submillimetre and $\mathrm{THz}$ waveguide components and circuits," Journal of Micromechanics and Microengineering, vol. 18, p. 095004, 2008.

[23] V. Desmaris, D. Meledin, D. Dochev, A. Pavolotsky, and V. Belitsky, "Terahertz components packaging using integrated waveguide technology," in Microwave Workshop Series on Millimeter Wave Integration Technologies (IMWS), 2011 IEEE MTT-S International, 2011, pp. 81-84.

[24] G. Chattopadhyay, J. S. Ward, H. Manohara, and R. Toda, "Deep Reactive Ion Etching based silicon micromachined components at terahertz frequencies for space applications," in Infrared, Millimeter and Terahertz Waves, 2008. IRMMW-THz. 2008. 33rd International Conference on, 2008, pp. 1-2.

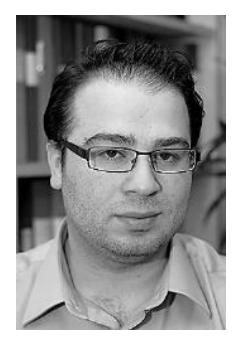

Hawal Rashid received the M.Sc. degree in engineering physics from the Chalmers University of Technology, Gothenburg, Sweden, 2010, and is currently working toward Ph.D. degree. His research interests are in low-noise cryogenic and room temperature $\mathrm{THz}$ systems, $\mathrm{THz}$ receiver component design, characterization and calibration.

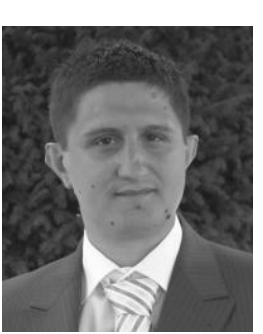

Vincent Desmaris received the M.Sc. degree in material science from the National Institute of Applied Science, Lyon, France, in 1999, and the Ph.D. degree in electrical engineering from the Chalmers University of Technology, Gothenburg, Sweden, in 2006. His thesis concerned the fabrication, characterization, and modeling of AlGaN/GaN microwave transistors. Since 2006, he has been with the Group for Advanced Receiver Development (GARD), Chalmers University of Technology, Göteborg, Sweden. His research interests are in the area of terahertz receiver technology, and especially mirofabrication and characterization of waveguide components and circuits, as well as planar cryogenic microwave devices

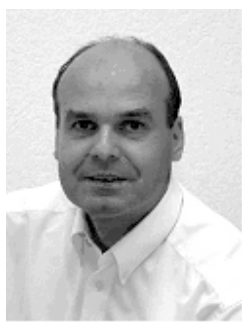

Thilo Bednorz received his master's degree in electrical engineering from the Technical University of Munich. From 1990 to 1993, he worked as design engineer in the spectrum analyzer group of Rohde \& Schwarz. 1994 he joined the network analyzer group when it was newly founded and works there as application engineer. 


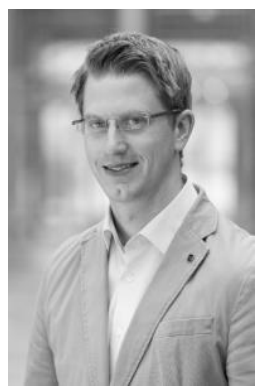

Marcel Ruf received the Dipl.-Ing. and Ph.D. degrees in electrical engineering from the Friedrich-Alexander-University of ErlangenNuremberg, Erlangen, Germany, in 2006 and 2013, respectively. From October 2006, he was with the Institute of Microwaves and Photonics, University of Erlangen-Nuremberg. In May 2013 he joined the network analysis development team of Rohde \& Schwarz GmbH \& Co. KG, Munich, Germany. His research interests include the development and fabrication of submillimeter wavelength multipliers and mixers, network analysis at millimeter-wave frequencies and beam instrumentation for medical linear electron accelerators

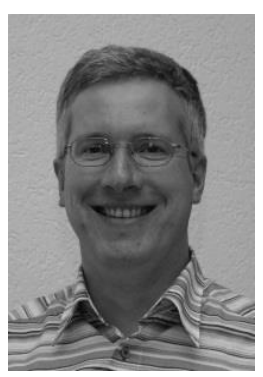

Andreas Henkel received the Dipl. Ing. degree in engineering from University of Siegen in Germany in 1995. He started as support engineer in Rohde \& Schwarz Engineering \& Sales GmbH, covering spectrum analyzers from Advantest. He then became product manager for optical test equipment and vector network analyzers from Advantest. In 2005 he became product manager for Rohde \& Schwarz vector network analyzers worldwide.

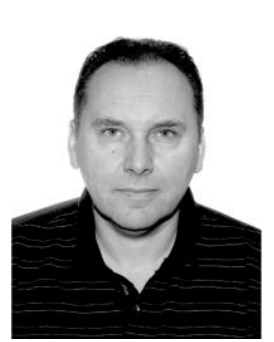

Victor Belitsky (M'95-SM'07) received the M.Sc. degree from the Moscow Telecommunication Institute, Moscow, Russia, in 1977, and the Ph.D. degree in experimental physics from the Institute of Radio Engineering and Electronics, U.S.S.R. Academy of Sciences, Moscow, Russia, in 1990. He is currently a Professor and leader of the Group for Advanced Receiver Development (GARD) at the Department of Earth and Space Sciences, Chalmers University of Technology, Gothenburg, Sweden. His research interests include $\mathrm{THz}$ electronics and components, instrumentation for radio astronomy and environmental science. 